\title{
A Research on Nitinol Alloy Material Fatigue Behavior Analysis of Cardiovascular Stent in Medical Engineering
}

\author{
Ö. KARAÇALI*
}

Department of Mechanical Engineering, Faculty of Engineering, Istanbul University, Avc1lar, Istanbul, 34320 Turkey

\begin{abstract}
Biocompatible cardiovascular stents are small cylindrical support structures introduced into the stenosed arteries to reopen the lumen and to restore blood flow in treating heart disease, which have revolutionized interventional cardiology. Cardiovascular stent designers are confronted with two basic requirements, such as an "infinite" life and the "thinnest" wires. Pulsatile pressure, repetitive mechanical forces, within the coronary artery may result in stent fatigue and fracture after stent implantation, particularly in patients with complex coronary disease. This research describes the simulation analysis of cardiovascular stents, to provide designers with estimates of their in vivo structural behavior and fatigue properties. Stent material failure or device fatigue remains major concern for stent manufactures and researchers. The objective of this research was to simulate the mechanical behavior of the stent using finite element method. A finite element analysis (FEA) of cardiovascular stent under fatigue cyclic loading conditions is presented. Commercial software was employed to study the fatigue performance of nitinol alloy materials in new stent systems. The effects of deployment, and static cyclic pressure loading on cardiovascular stent fatigue life were simulated and analyzed for nitinol alloy material. The investigation results displayed a significant correlation between material combinations, stent loading, and fatigue behavior.
\end{abstract}

DOI: $10.12693 /$ APhysPolA.127.1167

PACS: 81.40.Np, 81.70.Bt, 81.05.Bx, 87.10.Kn

\section{Introduction}

A stent is a small biocompatible implant, coiled wiremesh tube that can be deployed into an artery and expanded percutaneously, using a catheter during angioplasty, to open a narrowed artery. The objective of this work was to evaluate the capability of a fracture mechanics life prediction methodology, to predict the performance of nitinol stent material in fatigue laboratory experiments. Nickel titanium, also known as nitinol, is a metal alloy of nickel and titanium, where the two elements are present in roughly equal atomic percentages [1]. Nitinol is becoming more and more popular in cardiovascular stent applications due to its remarkable super elasticity, shape memory, biocompatibility, corrosion resistance, as well as fatigue resistance and durability $[2,3]$. In this research, a computational analysis [4] of stent material and its impact on mechanical characteristics under cyclic pressure loads was carried out by employing the finite element analysis.

Nitinol alloy has shape-memory property and high recoverable strain-super elasticity [4]. Such characteristics derive from the austenite to martensitic phase transformation, resulting from the near equi-atomic nickel and titanium content of the alloy, depicted by the stress hysteresis in Fig. 1. The maximum strain recovery after plastic deformation is $8.5 \%$ [5]. The applied radial and outward forces of expanding stent wire and the interaction with the blood vessel are depicted in Fig. 2.

\footnotetext{
*e-mail: ozdogank@istanbul.edu.tr
}

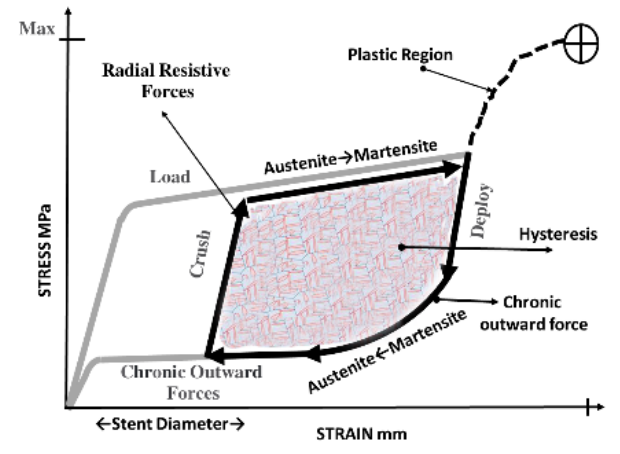

Fig. 1. Stress-strain curve for stent material of nitinol.

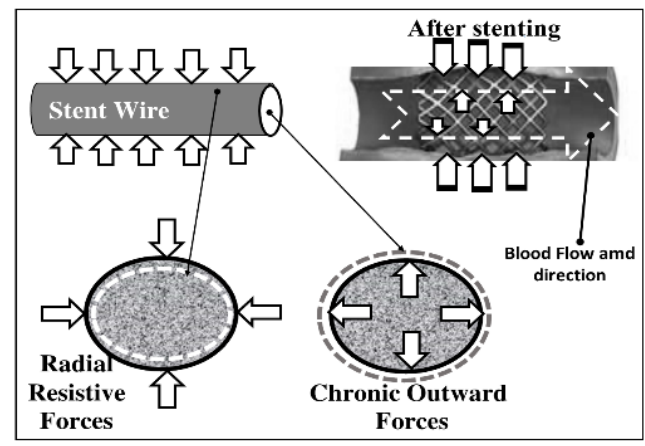

Fig. 2. Radial and outward forces in stent wire.

\section{Material and method}

The realization of design, manufacturing, and testing of a stent requires $\mathrm{CAD} / \mathrm{CAM} / \mathrm{CAE}$ software. Computer aided tools were used to evaluate the stent material's performance and to predict the stent's long-term fatigue life. Three steps are taken into account for designing a 
better stent implant, that satisfies the material requirements in this research: (1) a fatigue test environment is developed and a fatigue testing for a stent is realized in laboratory environment, (2) distribution of Von Mises stress [5] and maximum values, for simulation of the stent material fatigue, were investigated by FEA model, developed to compare stress-strain of nitinol stent, (3) metal fatigue quantitative study of the Goodman diagram, recommended by the FDA for stent analysis $[6,7]$. Equation of the Goodman diagram is given in the text below to quantify the interaction of mean and alternating stresses on the fatigue life of a material.

The flexural test method covered the determination of the durability of a stent by exposing it to physiologically relevant diametric distension levels by means of cyclic loading in the laboratory. This testing was carried out on a stent test specimen that was deployed into an elastically simulated vessel. The equivalent duration of this test is 20 years of use (at 72 beats per minute), or 980 million cycles. For measurements of tensile properties an Instron Tensile Tester (5500 series) was employed. Tensile properties of nitinol stent were evaluated by using ASTM F2516, which specifies the method to understand the upper and lower plateau strengths, tensile strength and elongation of the super elastic nitinol material. Fatigue testing and analysis was conducted according to the ISO standards 25539-2:2008, Part 2.

The 3D geometry of the repeatable units of the stents was generated using SOLIDWORKS Software. Using the repeating unit geometry of each stent design, solid models were generated, as shown in Fig. 3. The stent designs were analyzed with nitinol. The material properties of nitinol are, Young's modulus - $83 \mathrm{GPa}$, Poisson's ratio - 0.32, Yield stress - $690 \mathrm{MPa}$. The material properties for the stent designs were assumed to be isotropic and linear elastic. FEA is used for evaluation of the stent acute performance and for prediction of the stent longterm fatigue life.

Von Mises stress mathematical equation was used in this study to check whether the design will withstand a given load condition. Using this information, one can say that the design will fail, if the maximum value of Von Mises stress induced in the material is larger than the strength of the material. It works well for most of the cases, especially when the material is ductile, like e.g. nitinol. Figure 3 shows the Von Mises stress contours in the artery, resulting from contact with the stent.

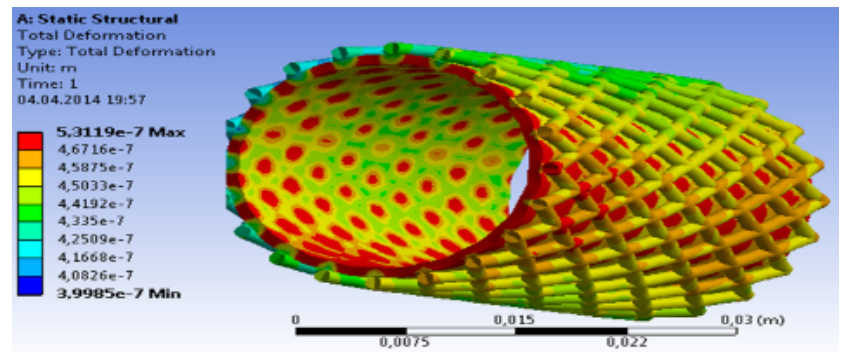

Fig. 3. Total deformation under static cycling load.

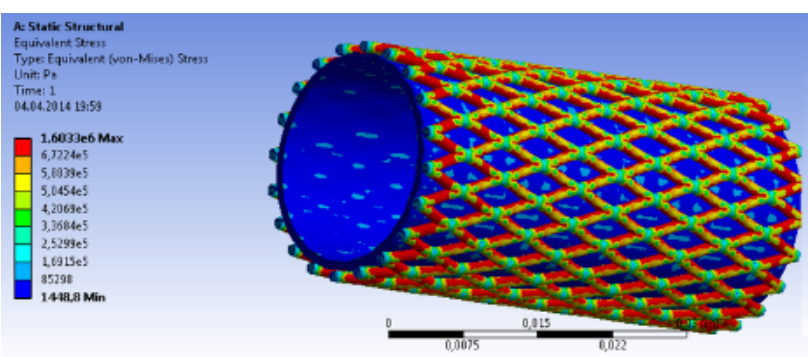

Fig. 4. Von Mises stress distribution by FEA model.

The stress concentrations in the contact region are due to artery's large deformations. It was also found that the maximum Von Mises stress of the arterial wall is the yield stress of 1.672 Mpa. As expected, the highest Von Mises stress occurs in the diamond cell apex as demonstrated in Fig. 4, due to the severe local curvatures. Because of the support via the stent, the struts experience a relatively low wall stress near the contact area.

The FEA enables calculation of circumferential and von Mises stress without incurring the expense of a stent. The finite-element material model used in the stent simulation was a Von Mises yield-criterion elastic-plastic model using the LS-DYNA [7] element, given in Fig. 5. It was found that the alternating strain in the stent under the pulse pressure of $50-150 \mathrm{mmHg}$ is $0.199 \%$. A safety factor of 1.9805 was deduced from the data. Figure 5 indicates that the highest risk of fatigue fracture is located at the strut's internal tensile side, close to the diamond connection. It is important to note the reason for a positive mean strain, even though the stent is under compression. The nitinol is modeled as a homogeneous, isotropic, elasto-plastic material through a Von Mises plasticity model. The percentage difference of Von Mises stresses between the finest and selected meshes is of $0.3 \%$. The artery mesh density is increased from 8050 to 340,076 elements. No appreciable difference in Von Mises stresses between the finest and the selected meshes is observed.

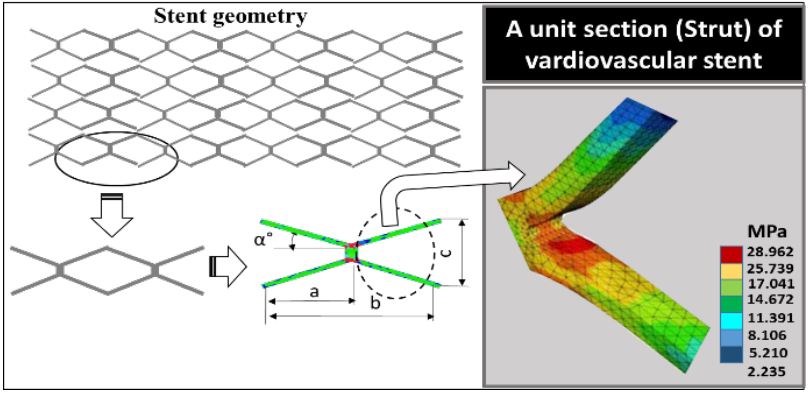

Fig. 5. Von Mises stress distribution after deployment.

To evaluate the stent long-term (over 20 years) fatigue performance under the loading conditions imposed by the systolic and diastolic arterial blood pressure loading, a Goodman fatigue analysis [8] was performed using the multi-axial stress. Because of the application of an alternating and reversed stresses, applied to cardiovascular nitinol stent, Goodman's formula proved to be the best 
suited analytical tool for the calculation of the equivalent stress. Goodman diagram is a graph of mean stress vs. alternating stress, showing when the material fails at some given number of cycles. Although Goodman analysis can provide factors of safety for highly loaded points in a stent, these apply for a single fatigue life only. The modified Goodman equation is defined by formula [6]:

$$
\sigma_{a}=\sigma_{e}\left(1-\frac{\sigma_{m}}{\sigma_{T S}}\right),
$$

where $\sigma_{a}$ is the alternating stress, $\sigma_{e}$ is the fatigue limit for completely reversed loading, $\sigma_{m}$ is the mean stress of loading, $\sigma_{T S}$ is the ultimate tensile strength, taken at $800 \mathrm{MPa}$, the saturated stress of constitutive model. A common way of assessing the fatigue life of alloys with regard to alternating strain amplitude and the mean strain on the designs, is to construct a Goodman diagram. The Goodman relationship is linear and the greater the mean strain the lower the alternating strain, required for fatigue failure. Current interpretations of the fatigue results do not take into account the material response change as a function of cycles and loading conditions. Rather, they are all based upon nonlinear FEA, that simulates the static loading and unloading on the stent. If the alternating strain is less than $0.4 \%$, the fatigue life is said to be infinite [8, 9]. For the cyclic loading, the mean strain and alternating strain were calculated for the entire element set using the ANSYS Goodman for fatigue analysis. As mentioned earlier, alternating strain had a greater effect on fatigue life than the mean strain and stress. A Goodman plot of the cardiac loading and blood pressure loading coupled with leaflet force loading is shown in Fig. 6.

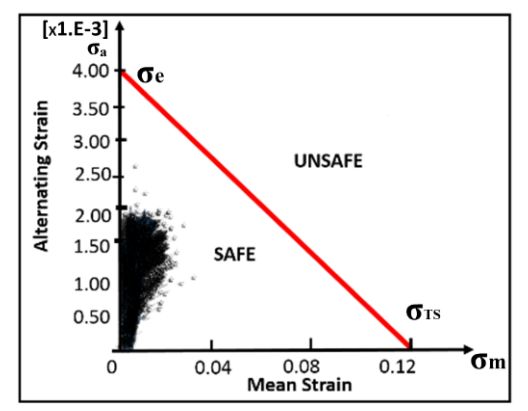

Fig. 6. Goodman analysis after stent fatigue deployment.

Calculated data are shown below the Goodman diagram failure line, indicating that the studied new stent was able to pass the fatigue life of $8.23 \times 10^{8}$ cycles under such combined loading conditions. Alternating diastolic to systolic pressures is applied to the vessel wall and the stress history is extracted for all regions of the finite element stent model for fatigue evaluation on a modified Goodman diagram. Additionally, the vessel is articulated resulting in bending, extension/compression, and twisting of the stented artery, and the fatigue life prediction of the stent is repeated. The cyclic load applied to the external surface of the stents resulted in the Goodman diagrams of Fig. 6. All points fell below the Goodman line. Points (362.48 MPa, 117.34 MPa) and (329.57 MPa, $110.39 \mathrm{MPa})$ were the closest to the curve and represented the worst-case fatigue area.

\section{Conclusions}

This research was aimed at understanding the effects of material characteristics, and fatigue behavior of nitinol on the mechanical properties of biocompatible selfexpanding stents. From the analysis of the experimental results, it may be concluded that the outcome of a real fatigue test was identical to the computational simulation data. The finite-element material model Von Mises yield criterion elastic-plastic model (using the LS-DYNA element) developed. Nitinol stent fatigue data was collected from a diamond-shaped specimen that was cycled to a maximum life of $8.23 \times 10^{8}$ cycles. Resulting data are presented on modified Goodman diagram in order to define regions of acceptable device life. The results revealed a significant correlation between material combinations, stent cyclic loading, and stent fatigue.

\section{Acknowledgments}

This research was supported by Scientific Research Projects (BAP) of Istanbul University, Turkey, Project No: 24082

\section{References}

[1] C. Lally, F. Dolan, P.J. Prendergast, J. of Biomech. 38, 1574 (2005).

[2] S. Pursnani, F. Korley, Journal of Cardiovascular Intervention 5, 476 (2012).

[3] B. O'Brien, J. Stinson, W. Carroll, J. of the mech. behav. of biomed. mat. 1, 303 (2008).

[4] N. Li, H. Zhang, H. Ouyang, Finite Elem. Analy. and Des. 45, 468 (2009).

[5] Y.P. Kathuria, Mat. Scie. and Eng. A 417, 40 (2006).

[6] A. García, E. Peña, M.A. Martínez, J. of the mech. behav. of biomed. mat. 10, 166 (2012).

[7] K. Takashima, T. Kitou, K. Mori, K. Ikeuchi, Med. Eng. and Phys. 29, 326 (2007).

[8] E.M.K. Abad, D. Pasini, R. Cecere, J. of Biomech. 45, 1028 (2012).

[9] T. Connolley, D. Nash, J. Buffiere, F. Sharif, P. Edward, P. McHugh, Med. Eng. and Phys. 29, 1132 (2007). 